Leser der MMW können sich ab sofort mit allen Fragen zur Abrechnung und Praxisführung an Helmut Walbert, Facharzt für Allgemeinmedizin, Würzburg, wenden. Sie erreichen ihn jeden Donnerstag von 13 bis 15 Uhr unter der kostenlosen Rufnummer (0800) 2379830 oder per E-Mail: w@lbert.info.

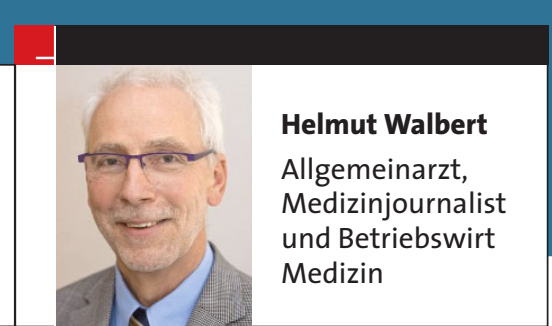

Mein Patient wurde im Krankenhaus behandelt

\title{
Jetzt will sein Arbeitgeber von mir einen AU-Schein
}

Dr. med. Gorski, Allgemeinarzt, KVWL: Ein Patient war wegen einer Unfallverletzung im Krankenhaus. Für die Zeit der stationären Behandlung erhielt er auf einem individuellen Vordruck der Klinik die entsprechende Bestätigung über die Zeit des stationären Aufenthaltes. Nach der Entlassung wurde inm von mir auf Vordruck Muster 1a die weitere Arbeitsunfähigkeit bestätigt. Sein Arbeitgeber schickt inn nun zu mir mit der Forderung, die Bestätigung des Krankenhauses auf Muster 1a zu bestätigen. Bin ich zu diesem Verwaltungsaufwand verpflichtet?"

Antwort: Nein! Als Vertragsarzt können und dürfen Sie nur ArbeitsunfähigkeitsBescheinigungen ausstellen für Zeiten der Arbeitsunfähigkeit, von denen Sie sich persönlich durch einen Arzt-Patienten-Kontakt von der Arbeitsunfähigkeit überzeugt haben. Die Richtlinie des Gemeinsamen Bundesausschusses lässt nur definierte Ausnahmen zu. Ansonsten gilt die in $\S 5$, Absatz 3 formulierte Bestimmung: ,Die Arbeitsunfähigkeit soll für eine vor der ersten Inanspruchnahme des Arztes liegende Zeit grundsätzlich nicht bescheinigt werden.“ Aus diesem Grund können Sie die Bestätigung des Krankenhauses nicht „umschreiben“. Der Arbeitgeber will offensichtlich den im Vertragsarztrecht vereinbarten „gelben“ Schein. Dieser ist gegebenenfalls vom Krankenhausarzt auszustellen. Sie sollten eine solche Bescheinigung auch deshalb unterlassen, weil sonst die Zeit des Krankenhausaufenthaltes in Ihrer Statistik über die von Ihnen zu verantwortenden Arbeitsunfähigkeitszeiten auftaucht.

Beachten Sie bitte auch die Regelungen für eine Rückdatierung: „Eine Rückdatierung des Beginns der Arbeitsunfähigkeit auf ei-

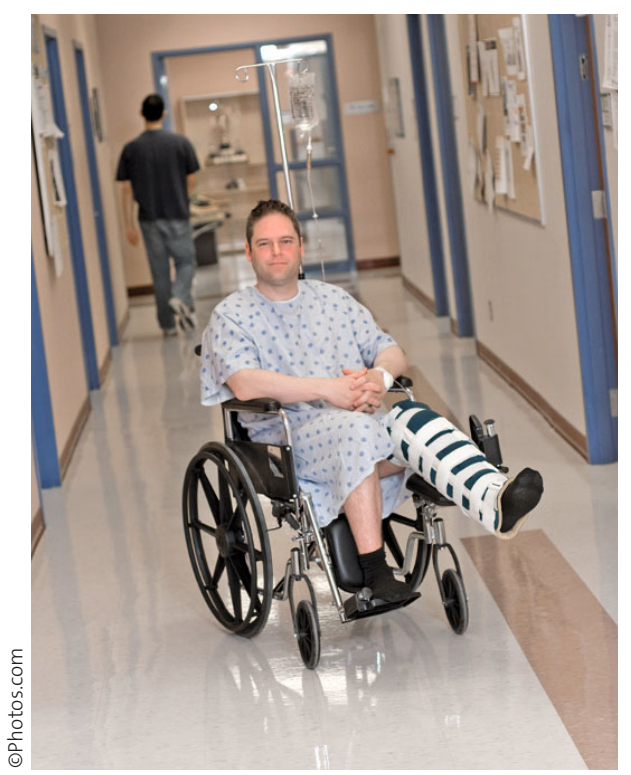

Ist ein Patient im Krankenhaus, erhält er den „gelben Schein" auch nur dort.

nen vor dem Behandlungsbeginn liegenden Tag ist ebenso wie eine rückwirkende Bescheinigung über das Fortbestehen der Arbeitsunfähigkeit nur ausnahmsweise und nur nach gewissenhafter Prüfung und in der Regel nur bis zu zwei Tagen zulässig."

\section{Gesundheitsfonds vor dem Verfassungsgericht}

Das Bundesverfassungsgericht in Karlsruhe hat die Klage eines HNO-Arztes gegen den Gesundheitsfonds angenommen. Damit ,ist eine wichtige Hürde genommen“, sagte der Beschwerdeführer Dr. Ulrich Brander, der in Brackenheim bei Heilbronn niedergelassen ist.

Brander reklamiert, er habe durch die Honorarreform und den Gesundheitsfonds hohe Verluste hinnehmen müssen. Nach eigenen Angaben erzielt er mit der Betreuung von rund 800 Patienten in seiner Landarztpraxis einen Umsatz von 6000 Euro monatlich. Das reiche lediglich, um die Praxiskosten zu refinanzieren, sagte Brander. Verdient habe er selber „praktisch nichts“. Vor allem wendet er sich dagegen, dass durch den Gesundheitsfonds Geld in andere Regionen abfließt, das für die Versorgung im Südwesten nicht mehr zur Verfügung steht.
Bei der Beschwerde, die der 1. Senat des Bundesverfassungsgerichts angenommen hat, handelt es sich um eine sogenannte Rechtssatz-Verfassungsbeschwerde, mit der sich der Arzt unmittelbar gegen das Gesetz wendet. In der Klagebegründung gibt Brander unter anderem an, in seiner Berufsfreiheit eingeschränkt zu werden, da der Gesetzgeber „berufsregelnd in unsere ärztliche Tätigkeit eingreift". $\quad$ FST . 